\title{
Regional versus systemic analgesia in video-assisted thoracoscopic lobectomy: a retrospective analysis
}

\author{
Benedikt Haager ${ }^{1}$, Daniel Schmid ${ }^{1,2}$, Joerg Eschbach², Bernward Passlick ${ }^{1}$ and Torsten Loop ${ }^{2 *}$ (D)
}

\begin{abstract}
Background: The optimal perioperative analgesic strategy in video-assisted thoracic surgery (VATS) for anatomic lung resections remains an open issue. Regional analgesic concepts as thoracic paravertebral or epidural analgesia were used as systemic opioid application. We hypothesized that regional anesthesia would provide improved analgesia compared to systemic analgesia with parenteral opioids in VATS lobectomy and would be associated with a lower incidence of pulmonary complications.
\end{abstract}

Methods: The study was approved by the local ethics committee (AZ 99/15) and registered (germanctr.de; DRKS00007529, 10th June 2015). A retrospective analysis of anesthetic and surgical records between July 2014 und February 2016 in a single university hospital with 103 who underwent VATS lobectomy. Comparison of regional anesthesia (i.e. thoracic paravertebral blockade (group TPVB) or thoracic epidural anesthesia (group TEA)) with a systemic opioid application (i.e. patient controlled analgesia (group PCA)). The primary endpoint was the postoperative pain level measured by Visual Analog Scale (VAS) at rest and during coughing during $120 \mathrm{~h}$. Secondary endpoints were postoperative pulmonary complications (i.e. atelectasis, pneumonia), hemodynamic variables and postoperative nausea and vomiting (PONV).

Results: Mean VAS values in rest or during coughing were measured below 3.5 in all groups showing effective analgesic therapy throughout the observation period. The VAS values at rest were comparable between all groups, VAS level during coughing in patients with PCA was higher but comparable except after 8-16 h postoperatively (PCA vs. TEA; $p<0.004$ ). There were no significant differences on secondary endpoints. Intraoperative Sufentanil consumption was significantly higher for patients without regional anesthesia $(p<0.0001$ vs. TPVB and vs. TEA). The morphine equivalence postoperatively applicated until POD 5 was comparable in all groups (mean \pm SD in mg: $32 \pm 29$ (TPVB), $30 \pm 27$ (TEA), $36 \pm 30$ (PCA); $p=0.6046$ ).

Conclusions: Analgesia with TEA, TPVB and PCA provided a comparable and effective pain relief after VATS anatomic resection without side effects. Our results indicate that PCA for VATS lobectomy may be a sufficient alternative compared to regional analgesia.

Trial registration: The study was registered (germanctr.de; DRKS00007529; 10th June, 2015).

Keywords: Minimal-invasive lung surgery, Thoracic paravertebral blockade, Thoracic epidural anesthesia, Patient controlled anesthesia

\footnotetext{
* Correspondence: torsten.loop@uniklinik-freiburg.de

${ }^{2}$ Department of Anesthesiology and Intensive Care Medicine, Medical Center,

University of Freiburg, Hugstetter Straße 55, 79106 Freiburg, Germany

Full list of author information is available at the end of the article
}

(c) The Author(s). 2019 Open Access This article is distributed under the terms of the Creative Commons Attribution 4.0 International License (http://creativecommons.org/licenses/by/4.0/), which permits unrestricted use, distribution, and reproduction in any medium, provided you give appropriate credit to the original author(s) and the source, provide a link to the Creative Commons license, and indicate if changes were made. The Creative Commons Public Domain Dedication waiver (http://creativecommons.org/publicdomain/zero/1.0/) applies to the data made available in this article, unless otherwise stated. 


\section{Background}

Video-assisted thoracic surgery (VATS) is considered as the standard minimal invasive surgical procedure for anatomic lung resections [1]. The advantages of VATS compared with open thoracotomy include faster recovery, reduced perioperative pain intensity, and decreased postoperative morbidity [2-4]. Nevertheless, persistent pain after VATS affects the ability to cough, impairs deep breathing and lung function, resulting in cardiorespiratory complications (> $15 \%)$, delayed recovery and increased costs [2].

The optimal perioperative analgesic strategy after VATS lobectomy remains contradictory. Thoracic epidural analgesia (TEA) is commonly considered as the gold standard for pain relief after open thoracotomy and is preferred by the majority of clinicians. In times of enhanced recovery and fast track concepts after surgery thoracic paravertebral block (TPVB) is an upcoming regional anesthesia technique in thoracic anesthesia. It can be used as single injection or continuous technique. However, there are also anesthesiologists in the field of thoracic anesthesia preferring patient-controlled analgesia (PCA) instead of the regional anesthesia techniques [5-7]. Regional analgesia techniques such as TEA may be not suitable for all patients for technical reasons or anticoagulative drug therapy and may be associated with numerous risks (e.g. dural perforation, spinal cord damage by formation of hematoma, infection and abscess; hypotension; urinary retention) $[8,9]$. The role of TPVB in this context has not been as clear but shown to be effective for pain relief with less hemodynamic side effects than TEA [5, 10-15]. As there is no evidence for one superior regional technique for pain relief after VATS, single-shot or continuous TPVB may be a suitable alternative to TEA or systemic opioid application. Intravenous patient-controlled analgesia with morphine analogue (PCA) is a widely used, simple, and convenient method [16].

After implementation of VATS as a standard for anatomic lung resection in our department the procedurespecific pain protocol was based on multimodal systemic analgesia with non-opioid and opioid drugs. Although less invasive, the thoracoscopic approach, resulted in unexpectedly high intensity of postoperative pain [17]. In this respect, regional analgesia (i.e. TEA or TPVB) was considered to be the crucial component of multimodal postoperative pain management in our department.

Accordingly, we wanted to analyze and undertook a retrospective analysis to establish whether thoracic regional analgesia (TPVB with ropivacaine alone or TEA) would provide improved analgesia compared with systemic analgesia with parenteral opioids and nonsteroidal analgesics leading to a reduction postoperative pulmonary complications such as atelectasis, pneumonia, hypoxia or pulmonary dysfunctions.

\section{Methods}

The study was approved by the local ethics committee (AZ 99/15) and registered (germanctr.de; DRKS00007529). Inclusion criteria were age older than 18 years and anatomic lung resection via VATS approach. Exclusion criteria were conversion to open thoracotomy, non-anatomic ("wedge") lung resections and additional chest wall resections. Data were retrospectively collected between July 2014 und February 2016. Patients signed a written informed consent approving their data could be used for scientific purposes. From July 2014 until January 2015 all patients received either a TPVB or a TEA. From January 2015 the perioperative analgesia was changed by interdisciplinary institutional decision and included systemic opioid application with piritramide as patient controlled analgesia (PCA; Graseby 3300; PCA Syringe Pump; SMITHS MEDICAL INTERNATIONAL LIMITED, Watford, Hertfordshire, United Kingdom).

\section{Anesthetic management}

The perioperative anesthetic regimen is standardized. Pre-medication before arrival in the operating room was performed with midazolam (3.75-7.5 mg p.o.). The responsible consultant preoperatively decided indication and choice for TPVB or TEA. Three experienced anesthesiologists performed all TEA and TPVB following the same protocol. Patients in the group TEA received an epidural catheter, using an $18 \mathrm{G}$ Tuohy needle, the epidural catheter (20G) will be placed at T4/5, T5/6, or T6/ 7 interspace (depending on the site of surgery) using the midline approach and hanging drop technique [18]. Epidural block analgesia was induced with $10 \mathrm{ml}$ of ropivacaine $0.2 \%$ and sufentanil $(0.2-0.3 \mu \mathrm{g} / \mathrm{kg}$, maximally $25 \mu \mathrm{g})$ administered as three separate injections, followed by a continuous infusion of ropivacaine $0.2 \%$ and sufentanil $0.5 \mu \mathrm{g} / \mathrm{ml}$ with a fixed infusion rate at $8 \mathrm{ml} / \mathrm{h}$ until $24 \mathrm{~h}$ after operation. The paravertebral space is located by using the technique described as previously described $[13,19]$. After introduction of the catheter $(3 \mathrm{~cm}$ into the paravertebral space), gentle aspiration, and test dose application ( $3 \mathrm{ml}$ of ropivacaine $0.5 \%$ with adrenalin $(5 \mu \mathrm{g} / \mathrm{ml}))$, thoracic paravertebral blockade (TPVB) was induced with $30 \mathrm{ml}$ ropivacaine $0.5 \%$ with adrenaline $(5 \mu \mathrm{g} / \mathrm{ml})$ followed by continuous paravertebral application of ropivacaine $0.2 \%$ (fixed infusion rate $8 \mathrm{ml} / \mathrm{h}$ ). The patients with systemic analgesia received an intravenous PCA, which was started immediately postoperative with a PCA device programmed to deliver piritramide i.v. (bolus dose of $1.5 \mathrm{mg}$, with a lockout time of $5 \mathrm{~min}$ and restricted total dose of $40 \mathrm{mg} / 4 \mathrm{~h}$ ). After an initial dose of $0.4-0.6 \mu \mathrm{g} / \mathrm{kg}$ of sufentanil, additional bolus doses of $0.1-0.2 \mu \mathrm{g} / \mathrm{kg}$ of sufentanil are administered as needed. Induction of anesthesia was performed by a targetcontrolled infusion (TCI) of with propofol (Propofol 1\% 
MCT \& Injectomat ${ }^{\circ}$ TIVA Agilia, Fresenius-Kabi GmbH, Bad Homburg, Germany) at plasma concentrations of 2-4 $\mu \mathrm{g}^{*} \mathrm{ml}^{-1}$. The Bispectral Index of the encephalogram (BIS) was monitored (BIS A-2000 monitor, Aspect Medical Systems, Newton, MA, USA) and the propofol concentration was decreased to $2.2 \mu \mathrm{g} / \mathrm{ml}$ at the lowest, if the BIS value decreases to below 30. Propofol concentration was increased to $4 \mu \mathrm{g}^{*} \mathrm{ml}^{-1}$ to avoid arterial blood pressure values above $20 \%$ of baseline and BIS values above 60 . Core temperature was kept above $36.0^{\circ} \mathrm{C}$ using a forced-air warming system. All patients were endotracheally intubated with a double-lumen endobronchial tube for one-lung ventilation.

\section{Surgical management}

VATS lobectomy was performed using a utility incision of $5 \mathrm{~cm}$ length entering the 4th intercostal space regardless which lobe was resected. Two further incisions of $1-2 \mathrm{~cm}$ were placed in posterior and anterior axillary line at level of the diaphragm in the 7 th or 8 th intercostal space. At the end of the procedure a 24 Fr chest tube was placed exiting the anterior lower incision in the 7 th or 8 th intercostal space. Following the same protocol two thoracic surgeons performed all procedures (B.P., B.H.). Chest tubes were removed when there was no air leakage for $6 \mathrm{~h}$ and the pleural fluid amount for $24 \mathrm{~h}$ did not exceed $200 \mathrm{ml}$.

\section{Postoperative pain management}

In the author's institution surgical patients were preoperatively instructed in the use of the visual analog scale (VAS). The VAS Score consisted of an unmarked $10 \mathrm{~cm}$ line, with $0 \mathrm{~cm}$ representing no pain and $10 \mathrm{~cm}$ the worst imaginable pain. Postoperatively all patients received a basic analgesic therapy containing either metamizole $4 \times 1 \mathrm{~g}$ per day or acetaminophen $3 \times 1 \mathrm{~g}$ depending on comorbidities (i.e. renal/liver dysfunction, allergies) and oxycodone $2 \times 20 \mathrm{mg}$ per day directly in the intermediate care unit. When the pain intensity exceeded $3 \mathrm{~cm}$ a bolus of piritramide $1.5 \mathrm{mg}$ was applicated and repeated until the pain level decreased below 3 (VAS) again. Patients of the PCA group were directly connected to an i.v. PCA device, delivering piritramide bolus doses of $1.5 \mathrm{mg}$ with a lockout time of $5 \mathrm{~min}$ and a total dose of $40 \mathrm{mg}$ in $4 \mathrm{~h}$. Nursing staff assessed pain intensity on the intermediate care unit in intervals of 4 $\mathrm{h}$ until $24 \mathrm{~h}$ after surgery, following once a day until 5 th postoperative day. Morphine equianalgesic conversion was calculated using the calculator based on the American Pain Society guideline (http://americanpainsociety.org/uploads/education/PAMI_Pain_Mangement_and_Dosing_Guide_02282017.pdf).

\section{Postoperative non-pain management}

Heart rate and arterial blood pressure were monitored continuously for the first $24 \mathrm{~h}$ postoperatively on the intermediate care unit and every $8 \mathrm{~h}$ after discharge to the ward. Hypotension was defined by mean arterial pressure below $60 \mathrm{mmHg}$. A chest $\mathrm{x}$-ray was routinely performed immediately after the operation and on the day following the removal of the chest tube. Radiologic infiltrates were defined by the written result from a consultant of the department of radiology. Pneumonia was defined by either radiologic proven infiltrate with necessity of antibiotic treatment or microbiological proof of bacteria making an antibiotic treatment necessary. A blood cell count was routinely performed on POD 1, leukocytosis was observed when leucocyte count exceeded $9.800 / \mathrm{ml}$. Pruritus, postoperative nausea and vomiting (PONV) and paresthesia were checked twice daily during the morning and afternoon round of the medical staff on the intensive care unit.

\section{Outcome measures}

Primary endpoint was the postoperative pain intensity assessed by the VAS in $\mathrm{cm}$ at different times after the procedure. VAS scores (0-10) were assessed by the nursing staff at the beginning of the shift routinely at rest and during coughing on ICU. When transferred to the ward after $24 \mathrm{~h}$, pain scores were documented once daily during the morning round of the nursing staff. Secondary outcome parameters were pulmonary (i.e. atelectasis, pneumonia, pulmonary embolism and respiratory failure) and surgical complications (i.e. leukocytosis, time to chest tube removal, pleural effusion) as well as side effects of the analgesic therapy (hypotension, pruritus, paresthesia, PONV). Atelectasis was defined by radiological criteria, pneumonia as fever, radiologic infiltration, positive microbiology or leukocytosis requiring antibiotic treatment.

\section{Statistical analyses}

Data were presented as mean and standard deviation ( \pm SD) or median and IQR if not indicated otherwise. Patient characteristic data were compared by analysis of variance (ANOVA) for multiple comparisons with Tukey post-hoc test. Comparisons of serial measurements (VAS for pain) were performed with repeated-measures ANOVA. Ranked data were analyzed with the KruskalWallis and Mann-Whitney U-tests when appropriate. Categorical data were examined by Fisher's exact or Chisquare test. Probability values under 0.05 were considered significant.

\section{Results}

From July 2014 to February 2016, 103 patients who underwent VATS lobectomy for oncologic reasons were examined retrospectively and initially included. In 62 patients 
analgesia was performed by regional anesthesia (28 patients with TPVB and 34 with TEA). From May 2015 patients were scheduled without regional anesthesia due to change in local procedures and 41 patients underwent VATS lobectomy with systemic opioid-based analgesia (PCA). Four patients were excluded due to conversion to systemic analgesia because of postoperative catheter dislocation, inadequate data sheet or open thoracotomy. The patient's demographic data are described in Table 1.

Mean VAS score was measured below 3.5 in all groups showing effective perioperative analgesia (Figs. 1 and 2). The VAS values at rest were comparable between all groups, VAS values during coughing were also effective and comparable with except higher in patients with PCA compared to TEA after $16 \mathrm{~h}$ postoperatively (Fig. 2). The intraoperative dose of sufentanil was significantly higher in the PCA group (Fig. 3; $p<0.0001$; mean dose $67 \pm$ $4 \mu \mathrm{g}$ for the PCA group vs. $47 \pm 3 \mu \mathrm{g}$ vs. patients with TPVB and $34 \pm 2$ vs. patients with TEA). The postoperative morphine equivalence dose applicated postoperatively until postoperative day 5 was comparable in all groups (Fig. 3: median (25-75\%) in mg: 25 (15-51) (TPVB), 20 (11-52) (TEA), 24 (16-51) (PCA); $p=0.60$ ).

Secondary endpoints showed no difference between the three groups (Table 2). Hemodynamic complications like hypotension demanding a vasopressor therapy were observed in 4 patients each with TPVB and PCA compared to 7 patients with TEA (Table 2). There was no pulmonary complication such as pulmonary embolism or respiratory failure. Pneumonia was diagnosed in 3 patients with TPVB, two patients with TEA, and 6 patients in the PCA group (Table 2). Pruritus was observed in one patient with TPVB and in 3 with TEA. No patient with PCA had pruritus (Table 2).

\section{Discussion}

This retrospective study analyzed two regional analgesic concepts (TPVB and TEA) and one systemic concept via opioid application using a PCA for postoperative analgesia in patients undergoing VATS lobectomy or VATS anatomic resections. The main findings can be summarized as follows: (i) TPVB, TEA and systemic analgesia provided effective analgesia $(\mathrm{VAS}<4$ ) during the perioperative period; (ii) the postoperative pain relief was comparable with similar opioid doses in all groups with the exception of $16 \mathrm{~h}$ postoperative while coughing favoring regional anesthesia; (iii) secondary outcome measures as respiratory or surgical complications did not differ among the three groups.

There are two major issues influencing the postoperative fast track concept in thoracic surgery demanding an optimal pain relief. First, early effective pain relief augments early mobilization with possible reduction of pulmonary complications (e.g. atelectasis, pneumonia) leading to early discharge and reduced health costs [20]. Secondly, thoracic surgery (especially thoracotomy) is associated with one of the highest incidences of chronic pain syndrome (up to 50\%) [21]. There was a significantly lower incidence for VATS procedures, though still a number of patients (34\%) emerge from VATS thoracic surgery suffering from chronic pain [22]. Placement of trocars and utility incision can cause intercostal nerve injury and pleural irritation as thoracotomy does, promoting pain transmission to the central nervous system leading to pain memory. Effective block of neural afferents can reduce acute postoperative pain and avoid the development of a pain consciousness [18].

For VATS resections the reports on different analgesic strategies are still very heterogeneous [7, 23, 24]. TEA is established as gold standard for thoracotomy and is

Table 1 Patient and surgical characteristics

\begin{tabular}{|c|c|c|c|c|c|}
\hline & $\operatorname{TPVB}(n=25)$ & TEA $(n=31)$ & PCA $(n=41)$ & Total $(n=97)$ & $P$ value \\
\hline \multicolumn{6}{|l|}{ Sex } \\
\hline female & 7 & 9 & $22^{*}$ & 38 & $P<0.04$ \\
\hline male & 18 & 22 & 19 & 59 & \\
\hline Age (median/range) (yr) & $69(45-81)$ & $72(46-88)$ & $68(43-81)$ & $70(43-88)$ & 0.865 \\
\hline ASA Score (n) & & & & & 0.520 \\
\hline I & 0 & 0 & 0 & 0 & \\
\hline$\|$ & 4 & 1 & 9 & 14 & \\
\hline III & 20 & 29 & 31 & 80 & \\
\hline IV & 1 & 1 & 1 & 3 & \\
\hline Type of surgery & & & & & 0.225 \\
\hline Lobectomy & 20 & 24 & 29 & 73 & \\
\hline Segment resection & 3 & 6 & 12 & 21 & \\
\hline Pneumonectomy & 2 & 1 & 0 & 3 & \\
\hline
\end{tabular}

TPVB Thoracic paravertebral blockade, TEA Thoracic epidural analgesia, PCA Patient controlled analgesia, ASA American Society of Anesthesiology; ${ }^{*} p$-value $<0.05$ 


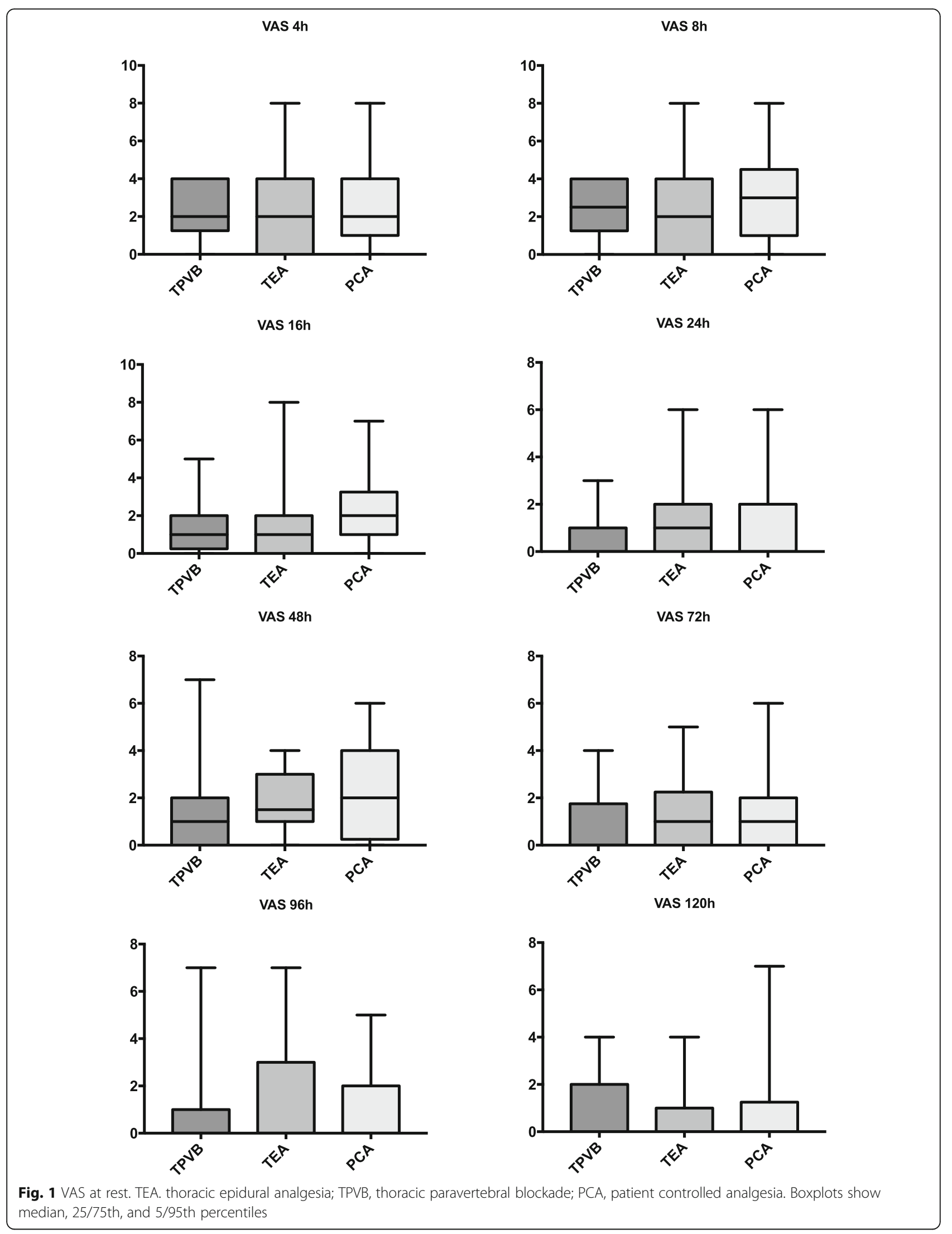




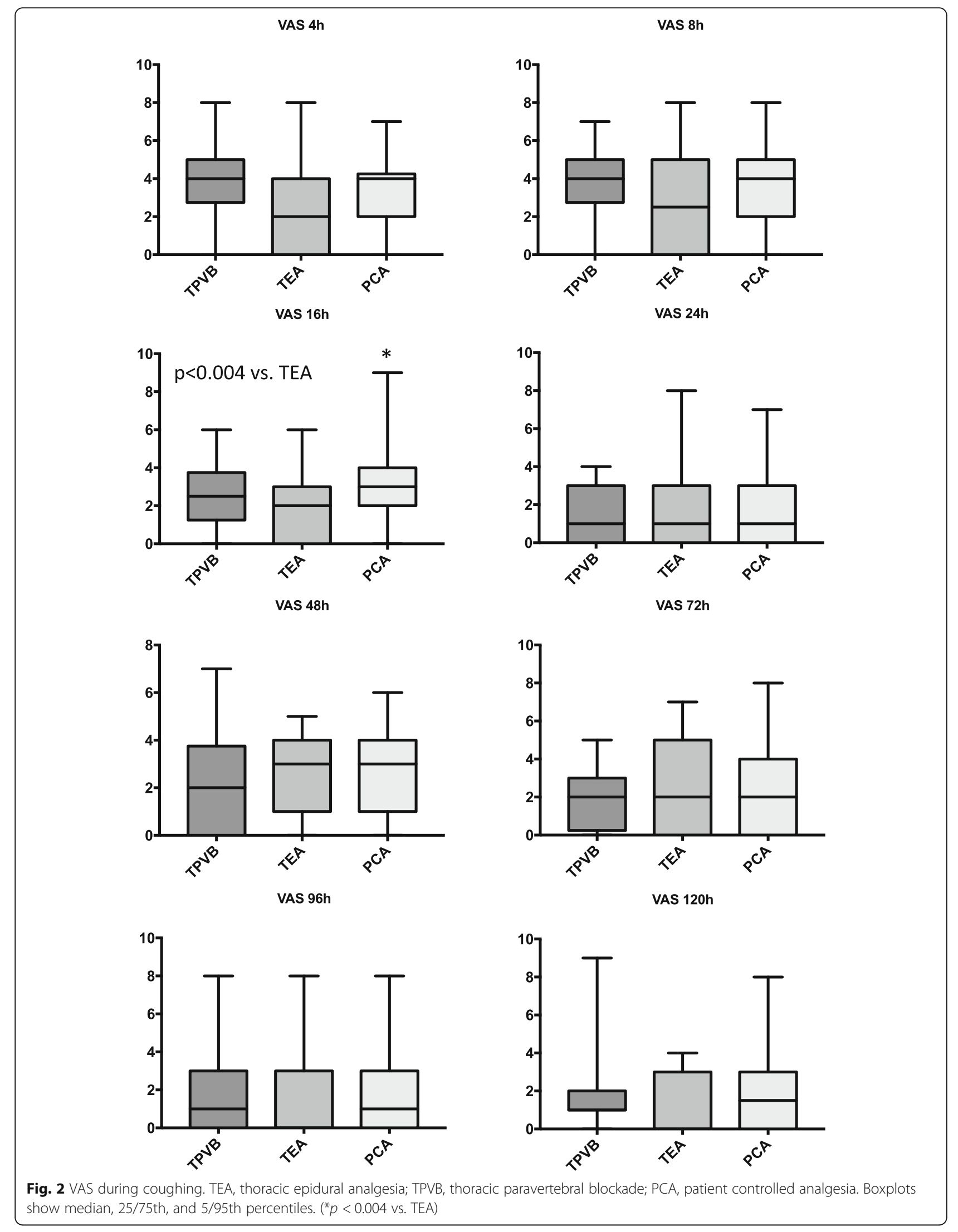




\section{Sufentanil intraoperatively}

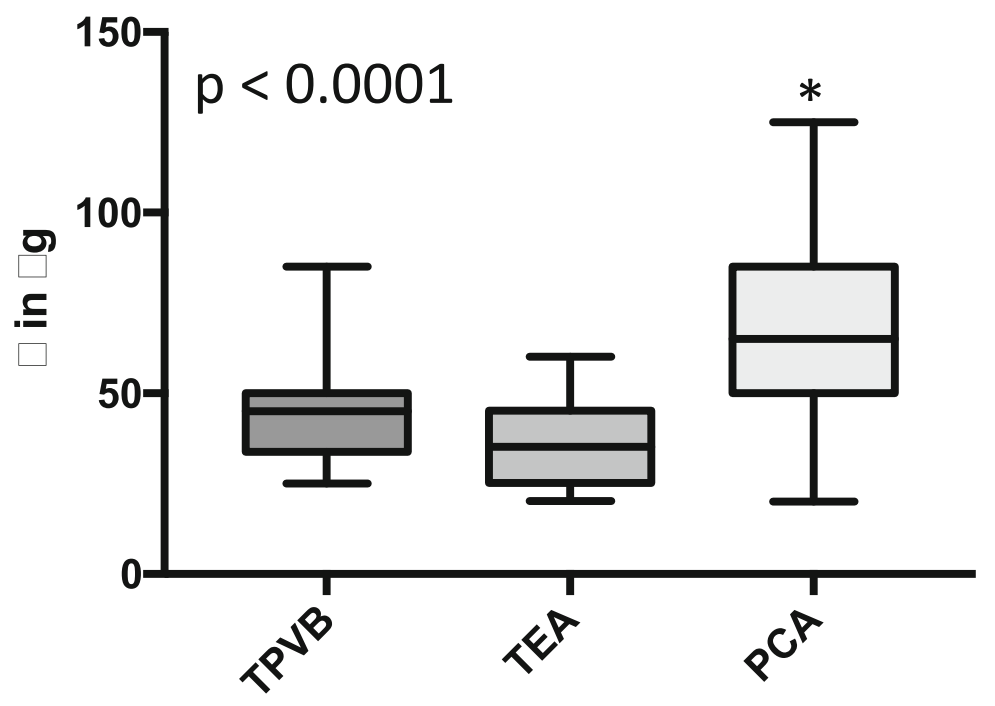

\section{Morphine Equivalence postoperatively}

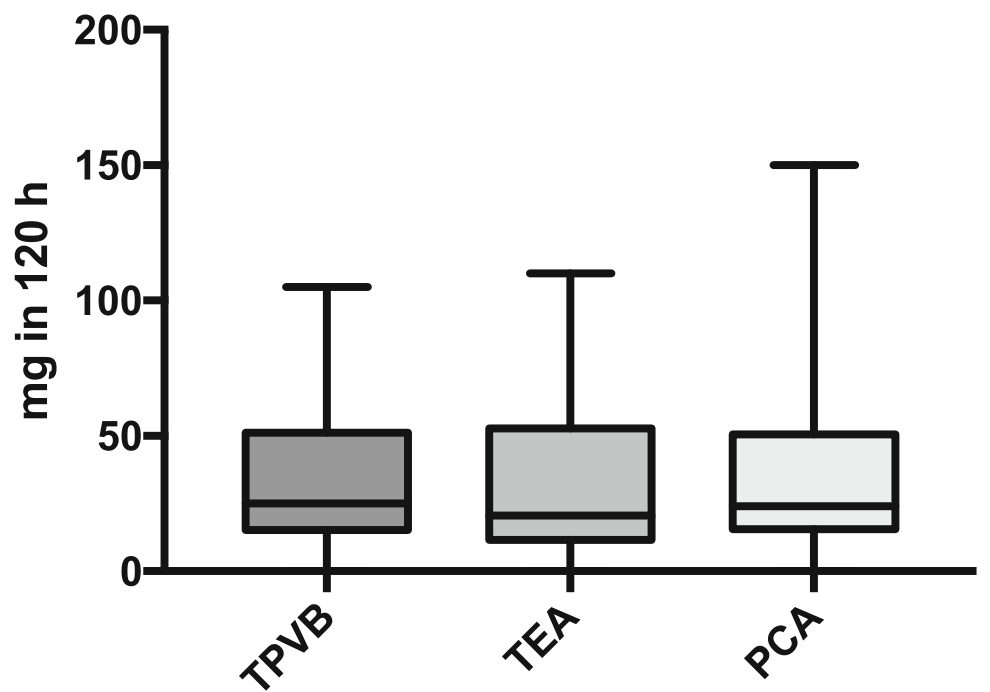

Fig. 3 Opioid consumption. TEA, thoracic epidural analgesia; TPVB, thoracic paravertebral blockade; PCA, patient controlled analgesia. Boxplots show median, $25 / 75$ th, and 5/95th percentiles. $\left({ }^{*} p<0.0001\right.$ vs. TEA and TPVB)

widely used for anatomic VATS resections as well as intravenous delivery of opioids via PCA [25-27]. The results of meta-analyses and reviews demonstrated that TPVB with local anesthetics has a comparable efficacy and a higher safety profile [14, 28, 29]. Kosinski et al. compared continuous epidural with paravertebral analgesia in a prospective randomized study and demonstrated a favorable effect for the paravertebral block on pain scores on the POD 1 and 2. The consumption of opioids was comparable and the authors found a higher rate of side effects for example urinary retention and hypotension in the TEA group. Beyond that the use of paravertebral block was recommended due to the better safety profile and comparable analgesic effect [30].

In this study, TEA, TPVB, and PCA provided a comparable pain relief. Two studies compared TEA with systemic opioid analgesia for thoracoscopic lobectomy. Kim et al. demonstrated in a non-blinded RCT of 37 patients that there were no differences in pain scores, supplementary analgesic requirements or adverse events [25]. 
Table 2 Secondary Endpoints

\begin{tabular}{llllll}
\hline & TPVB $(n=25)$ & TEA $(n=31)$ & PCA $(n=41)$ & Total $(n=97)$ & $P$ value \\
\hline Hemodynamics $(n)$ & & & & 15 & 0.445 \\
Hypotension & 4 & 7 & 4 & 35 & 86 \\
Hypertension & 23 & 28 & 6 & 11 & 0.976 \\
Pneumonia (n) & 3 & 2 & 0 & 4 & 0.617 \\
Pruritus (n) & 1 & 3 & 21 & 50 & 0.149 \\
Leucocytosis (n) & 16 & 13 & 0.653 \\
\hline
\end{tabular}

TPVB Thoracic paravertebral blockade, TEA Thoracic epidural analgesia, PCA Patient controlled analgesia

Yie et al. investigated 105 patients retrospectively and found a lower VAS in the TEA group, but only on POD 2. Incidence of dizziness was shown to be higher in the morphine group on POD 1, whereas pruritus was higher in the TEA group on POD 2 and 3 [26].

There were no differences with respect to the pain intensity between opioid PCA and TEA or TPVB. The cost implications of a regional analgesia concept, TEA or TPVB, compared with i.v. analgesia is well documented. The cost difference is mainly caused by the professional manpower costs and the treatment of complications [31].

In addition to the comparable pain relief we found no difference between the secondary outcomes (e.g. pulmonary complications, surgical complications, PONV) not supporting our hypothesis that there would be less pulmonary complications using a regional anesthetic procedure.

This study had some limitations. The trial was not a prospective, randomized, and double-blinded clinical study, but technique and expertise in TEA or TPVB was very homogeneous and performed exclusively by three consultants and reflects clinical practice. In addition, pain assessment was routinely evaluated only at the described time points. Secondly, the small sample size limited the possibility of drawing a definitive conclusion. Thirdly, the sample size of the study was not calculated due to the retrospective design and therefore to small to evaluate the secondary outcomes of respiratory function, pulmonary complications, nausea and vomiting, degree of sedation, hypotension, and pruritus. Finally, assessment of the level of analgesic effect or sensory block of the TEA and TPVB were not routinely performed.

\section{Conclusions}

In conclusion, our findings indicated that application of systemic opioids via PCA device was an effective and acceptable alternative to regional anesthesia with TEA or TPVB for postoperative pain relief for patients undergoing VATS lobectomy.

\section{Abbreviations}

ANOVA: Analysis of variance; PCA: Patient-controlled analgesia; POD: Postoperative day; PONV: Postoperative nausea and vomiting; TCl: Target-controlled infusion; TEA: Thoracic epidural analgesia;
TPVB: Thoracic paravertebral block; VAS: Visual analog scale; VATS: Videoassisted thoracic surgery

\section{Acknowledgements}

Not applicable.

\section{Authors' contributions}

$\mathrm{BH}$ initiated and designed the study, DS designed the study database and collected the case reports, JE is responsible for the acute pain service and the corresponding documentation. BP proofread the manuscript and contributed to the study design. TL performed statistical processing, writing and drafting of the manuscript. All authors read and approved the manuscript in its final version.

\section{Funding}

None of the authors received funding for contribution to this study.

\section{Availability of data and materials}

The datasets generated and analyzed during the current study are not publicly available due institutional restrictions but are available from the corresponding author on reasonable request.

\section{Ethics approval and consent to participate}

This study was approved by the local Ethics Committee University of Freiburg, Germany (approval number AZ 99/15; Ethik-Kommission der AlbertLudwigs-Universität Freiburg, Engelberger Straße 21, 79106 Freiburg). An individual consent of the patients was not required because all methods of analgesia are standard approachs in our institution. The study has a retrospective design, and only closed cases were enclosed. The institutional review board declined the need for retrospective information and consent of the analyzed patients.

\section{Consent for publication}

Not applicable.

\section{Competing interests}

The authors declare that they have no competing interests.

\section{Author details}

'Department of Thoracic Surgery, Medical Center, University of Freiburg, Hugstetter Straße 55, 79106 Freiburg, Germany. ${ }^{2}$ Department of Anesthesiology and Intensive Care Medicine, Medical Center, University of Freiburg, Hugstetter Straße 55, 79106 Freiburg, Germany.

Received: 27 August 2018 Accepted: 17 September 2019 Published online: 17 October 2019

\section{References}

1. Steinthorsdottir KJ, Wildgaard L, Hansen HJ, et al. Regional analgesia for video-assisted thoracic surgery: a systematic review. Eur I Cardiothorac Surg. 2014:45:959-66.

2. Falcoz PE, Puyraveau M, Thomas PA, et al. Video-assisted thoracoscopic surgery versus open lobectomy for primary non-small-cell lung cancer: a propensity-matched analysis of outcome from the European Society of Thoracic Surgeon database. Eur I Cardiothorac Surg. 2016;49:602-9.

3. Bendixen $\mathrm{M}$, Jorgensen $\mathrm{OD}$, Kronborg $\mathrm{C}$, et al. Postoperative pain and quality of life after lobectomy via video-assisted thoracoscopic surgery or 
anterolateral thoracotomy for early stage lung cancer: a randomised controlled trial. Lancet Oncol. 2016;17:836-44.

4. McKenna RJ Jr, Houck W, Fuller CB. Video-assisted thoracic surgery lobectomy: experience with 1,100 cases. Ann Thorac Surg. 2006;81:421-5.

5. Joshi GP, Bonnet $F$, Shah $R$, et al. A systematic review of randomized trials evaluating regional techniques for postthoracotomy analgesia. Anesth Analg. 2008;107:1026-40.

6. Wenk M, Schug SA. Perioperative pain management after thoracotomy. Curr Opin Anaesthesiol. 2011;24:8-12.

7. Shanthanna H, Moisuik P, O'Hare T, et al. Survey of postoperative regional analgesia for thoracoscopic surgeries in Canada. J Cardiothorac Vasc Anesth. 2018;32:1750-5.

8. Popping DM, Wenk M, Van Aken HK. Neurologic complications after epidural analgesia. AINS. 2012;47:336-43.

9. Horlocker $\Pi$. Regional anaesthesia in the patient receiving antithrombotic and antiplatelet therapy. Br J Anaesth. 2011;107(Suppl 1):196-106.

10. Messina M, Boroli F, Landoni G, et al. A comparison of epidural vs. paravertebral blockade in thoracic surgery. Minerva Anestesiol. 2009;75:616-21.

11. Thavaneswaran P, Rudkin GE, Cooter RD, et al. Brief reports: paravertebral block for anesthesia: a systematic review. Anesth Analg. 2010;110:1740-4.

12. Marret $E$, Bazelly B, Taylor $G$, et al. Paravertebral block with ropivacaine $0.5 \%$ versus systemic analgesia for pain relief after thoracotomy. Ann Thorac Surg. 2005:79:2109-13.

13. Karmakar MK. Thoracic paravertebral block. Anesthesiology. 2001;95:771-80.

14. Davies RG, Myles PS, Graham JM. A comparison of the analgesic efficacy and side-effects of paravertebral vs epidural blockade for thoracotomy--a systematic review and meta-analysis of randomized trials. $\mathrm{Br} J$ Anaesth. 2006;96:418-26.

15. Powell ES, Cook D, Pearce AC, et al. A prospective, multicentre, observational cohort study of analgesia and outcome after pneumonectomy. Br J Anaesth. 2011;106:364-70.

16. Hudcova J, McNicol E, Quah C, et al. Patient controlled opioid analgesia versus conventional opioid analgesia for postoperative pain. Cochrane Database Syst Rev. 2006:4:CD003348.

17. Dango S, Harris S, Offner K, et al. Combined paravertebral and intrathecal vs thoracic epidural analgesia for post-thoracotomy pain relief. Br J Anaesth. 2013;110:443-9.

18. Richardson J, Lonnqvist PA. Thoracic paravertebral block. Br J Anaesth. 1998 81:230-8.

19. Kehlet $H$, Dahl JB. Anaesthesia, surgery, and challenges in postoperative recovery. Lancet. 2003;362:1921-8.

20. Gottschalk A, Ochroch EA. Clinical and demographic characteristics of patients with chronic pain after major thoracotomy. Clin J Pain. 2008;24: 708-16.

21. Shanthanna H, Aboutouk D, Poon E, et al. A retrospective study of open thoracotomies versus thoracoscopic surgeries for persistent postthoracotomy pain. J Clin Anesth. 2016;35:215-20.

22. Hutchins J, Sanchez J, Andrade R, et al. Ultrasound-guided paravertebral catheter versus intercostal blocks for postoperative pain control in videoassisted thoracoscopic surgery: a prospective randomized trial. J Cardiothorac Vasc Anesth. 2017;31:458-63.

23. Duale C, Gayraud G, Taheri H, et al. A French Nationwide survey on anesthesiologist-perceived barriers to the use of epidural and paravertebral block in thoracic surgery. J Cardiothorac Vasc Anesth. 2015;29:942-9.

24. Kim JA, Kim TH, Yang M, et al. Is intravenous patient controlled analgesia enough for pain control in patients who underwent thoracoscopy? J Korean Med Sci. 2009;24:930-5.

25. Yie JC, Yang JT, Wu CY, et al. Patient-controlled analgesia (PCA) following video-assisted thoracoscopic lobectomy: comparison of epidural PCA and intravenous PCA. Acta Anaesthesiol Taiwanica. 2012;50:92-5.

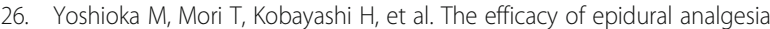
after video-assisted thoracoscopic surgery: a randomized control study. Ann Thorac Cardiovasc Surg. 2006;12:313-8.

27. Baidya DK, Khanna P, Maitra S. Analgesic efficacy and safety of thoracic paravertebral and epidural analgesia for thoracic surgery: a systematic review and meta-analysis. Interact Cardiovasc Thorac Surg. 2014;18:626-35.

28. Demmy TL, Nwogu C, Solan P, et al. Chest tube-delivered bupivacaine improves pain and decreases opioid use after thoracoscopy. Ann Thoracic Surg. 2009;87:1040-6.

29. Kosinski S, Fryzlewicz E, Wilkojc M, et al. Comparison of continuous epidura block and continuous paravertebral block in postoperative analgaesia after video-assisted thoracoscopic surgery lobectomy: a randomised, noninferiority trial. Anaesthesiol Intens Ther. 2016:48:280-7.

30. Macario A, Scibetta WC, Navarro J, et al. Analgesia for labor pain: a cost model. Anesthesiology. 2000:92:841-50

31. Bartha E, Carlsson P, Kalman S. Evaluation of costs and effects of epidural analgesia and patient-controlled intravenous analgesia after major abdominal surgery. Br J Anaesth. 2006;96:111-7.

\section{Publisher's Note}

Springer Nature remains neutral with regard to jurisdictional claims in published maps and institutional affiliations.
Ready to submit your research? Choose BMC and benefit from:

- fast, convenient online submission

- thorough peer review by experienced researchers in your field

- rapid publication on acceptance

- support for research data, including large and complex data types

- gold Open Access which fosters wider collaboration and increased citations

- maximum visibility for your research: over $100 \mathrm{M}$ website views per year

At BMC, research is always in progress.

Learn more biomedcentral.com/submissions 